\title{
The use of platelet-rich plasma (PRP) to treat chronic tendinopathies: a technical analysis
}

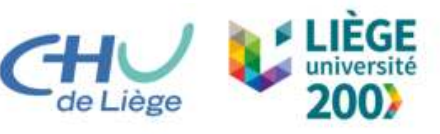

\section{Kaux JF, Emonds-Alt T}

Department of Physical Medicine and Sports Traumatology, SportS2,

FIFA Medical Centrer of Excellence, University and University Hospital of Liège, Belgium

1. Introduction: Platelet-rich plasma (PRP) is blood plasma with a high concentration of autologous platelets which constitute an immense reservoir of growth factors. The clinical use of PRP is widespread in various medical applications. Although highly popular with athletes, the use of PRP for the treatment of tendinopathies remains scientifically controversial, particularly due to the diversity of products that go by the name of "PRP". This may partly explain the disparity of results obtained in the literature.

2. Purpose: The objective of this study was to evaluate the similarities and differences between the variety of PRP formulations, preparation, and uses of this techniques in literature and to try to determine characteristics of the PRP which tend to give the best responses for the treatment of knee osteoarthritis.

3. Methods: A search for articles was conducted in the Pubmed and Pedro databases. The results were obtained by using the following key words and combinations of these key words: PRP, platelet-rich plasma, injection, treatment, tennis elbow, greater trochanteric pain syndrome, jumper's knee, Achilles tendinopathy, plantar fasciitis, anticoagulant, sodium chloride, local anesthetic, and centrifugation speed.

In this literature review, we take a closer look at eight parameters which may influence the quality of PRP: 1) anticoagulants used to preserve the best platelet function, 2 ) the speed of centrifugation used to extract the platelets, 3) the platelet concentrations obtained, 4) the impact of the concentration of red and while blood cells on PRP actions, 5) platelet activators encouraging platelet degranulation and, hence, the release of growth factors, and 6) the use or nonuse of local anesthetics when carrying out infiltration. In addition to these parameters, it may be interesting to analyze other variables such as 7) the use of ultrasound guidance during the injection with a view to determining the influence they have on potential recovery.
4. Results: Analysis of the 59 studies shows that a majority use ACDA as an anticoagulant during sampling. A platelet activator (calcium chloride) is only used in a few studies. However, the best results have been obtained in studies which use no platelet activator. In terms of the speed of centrifugation, analysis of all these studies appears to confirm the existence of a great number of protocols in the literature. It is difficult to draw any conclusions when these different variables are unknown (volume of initial sample, equipment used to obtain the PRP). Currently, no studies have been carried out neither on the use of a "buffer" product to neutralize the acidity caused by the anticoagulant nor on the optimal volume of PRP to be injected. Although it would appear inadvisable to administer a local anesthetic because the anesthetic may reduce the local $\mathrm{pH}$ and lead to the inhibition, reduction, or absence of platelet degranulation, the literature shows that the majority use a local anesthetic. They do not, however, obtain better results than those not using a local anesthetic. Finally, it appears to be advisable to carry out infiltration under ultrasound guidance, even if PRP diffusion is observed after injection. This all aims to show that there is still a need for high quality studies, with standardized collection protocols and the use of PRP in the context of tendinopathies, in order to better scientifically understand the real effectiveness.

5. Conclusions: Although there is an increasing amount of scientific proof about the benefits of PRP infiltration in the treatment of tendinopathies, there is currently no consensus as to how to obtain or use it. This may partly explain the disparity of results obtained in the literature. Similarly, the biological parameters and risk factors for tendinopathies of patients themselves may possibly influence the results of this treatment even if, to date, this has not yet been demonstrated. There is a lack of standardization in PRP preparation technique for chronic tendinopathies. However, our study helped identify features of PRP recommended a platelet concentration lower than 5 times the baseline (from 3 to 4 ), and avoiding leukocytes and erythrocytes. We recommend leveraging this information about PRP for future studies.

Kaux JF, Emonds-Alt T. The use of platelet-rich plasma to treat chronic tendinopathies: A technical analysis. Platelets. $2017 \mathrm{Jul}$ 31:1-15. [Epub ahead of print] 